\title{
BMJ Open Long-term psychosocial impact of venous thromboembolism: a qualitative study in the community
}

\author{
Rachael Hunter, ${ }^{1}$ Simon Noble, ${ }^{2}$ Sarah Lewis, ${ }^{3}$ Paul Bennett ${ }^{4}$
}

To cite: Hunter R, Noble S, Lewis S, et al. Long-term psychosocial impact of venous thromboembolism: a qualitative study in the community. BMJ Open 2019;9:e024805. doi:10.1136/ bmjopen-2018-024805

- Prepublication history for this paper is available online. To view these files, please visit the journal online (http://dx.doi. org/10.1136/bmjopen-2018024805).

Received 15 June 2018 Revised 10 December 2018 Accepted 20 December 2018

Check for updates

(C) Author(s) (or their employer(s)) 2019. Re-use permitted under CC BY-NC. No commercial re-use. See rights and permissions. Published by BMJ.

${ }^{1}$ Department of Psychology, Swansea University, Swansea, UK

${ }^{2}$ Department of Palliative Medicine, Cardiff University,

Newport, South Wales, UK

${ }^{3}$ Department of Haematology,

Aneurin Bevan University Health

Board, Abergavenny, UK

${ }^{4}$ Department of Clinical

Psychology, Swansea University, Swansea, UK

Correspondence to

Dr Rachael Hunter;

r.hunter@swansea.ac.uk

\section{ABSTRACT}

Objectives Venous thromboembolism (VTE) is a serious, potentially traumatic, life-threatening condition and a major cause of mortality and morbidity. The aim of this study was to obtain detailed understandings of the impact of VTE and examine individual's experiences over the first year since a first-time VTE.

Design A longitudinal qualitative interview study using inductive thematic analysis. This study presents followup data for 11 participants, first interviewed 6 months following a first-time VTE.

Setting Outpatients recruited from a community haematology clinic in a UK District General Hospital. Participants Eleven participants (seven females and four males) recruited from a community haematology clinic. Participants had experienced a first-time VTE and participated in qualitative interviews 3 months previously. Intervention Audio-recorded semistructured interviews with a sample of 11 participants who experienced a first-time deep vein thrombosis or pulmonary embolism within the previous year. Interviews were transcribed and analysed using inductive thematic analysis.

Results Four overarching themes were identified: life changing and forever changed, the trauma of care, 'thrombo-neuroses' and through adversity comes growth. Theme content varied according to age and developmental stage, presence of VTE symptoms and the experience of diagnosis.

Conclusions The data demonstrate the psychosocial impact of VTE and its diagnosis as physically and psychologically challenging, and individuals reported being forever changed by the experience. Participants' reported continued high levels of trauma and anxiety symptoms, triggered by physical (eg, symptoms) and psychological (eg, health anxiety, negative emotions) reminders of VTE. Wider primary care service issues including misdiagnosis maintained negative emotions and health anxiety with implications for relationships with professionals. Targeted clinical interventions to better identify and support individuals at risk of distress and enhance psychological well-being and reduce distress are discussed.

\section{INTRODUCTION}

Venous thromboembolism (VTE), comprising deep vein thrombosis (DVT) and pulmonary embolism (PE), has an incidence rate of 1-2 per 1000 persons in western populations. ${ }^{1}$ Mortality rates are high, ${ }^{2}$ and around $25 \%$

\section{Strengths and limitations of this study}

This is the first longitudinal study of psychosocial impact of venous thromboembolism.

- The study had sequential entry so is as representative as possible of people early in their patient career.

- The patients were recruited from a single site.

- This paper gives patients a voice and highlights unmet clinical needs.

- While appropriate in numbers for thematic analysis, the number of participants is relatively low so there must be caution in generalisation of findings.

of those affected experience further events over the subsequent 5 years. ${ }^{3}$ Post-thrombotic syndrome is a complicating factor in $25 \%$ to $50 \%$ of DVT cases, ${ }^{4}$ presenting a considerable burden for services and the individual.

Despite substantial research into its medical consequences, the emotional and psychological implications of VTE have received less attention. Studies ${ }^{5-7}$ have highlighted the impaired quality of life across physical, social and psychological domains in the early months following the event. However, no study has examined the evolving emotional and coping experiences in a representative sample of VTE patients over time. This is despite evidence that psychological distress among the chronically ill is associated with a range of adverse consequences, including reduced adherence treatment, increased symptom burden and elevated levels of disability and death. ${ }^{8}$

The present study addresses this gap. It follows up 11 participants, first interviewed during the first 6 months following a firsttime VTE. At this time, themes from inductive thematic analysis included VTE as a life changing and traumatic experience; living with uncertainty and fear of reoccurrence; feeling let down by health services, and positive changes and outcomes. Thematic content varied according to participants' age 


\begin{tabular}{lllll}
\hline Table 1 & Participant characteristics & & & \\
\hline Pt. No & Pseudonym & Age (years) & VTE event & Relevant information \\
\hline 1 & Fiona & 24 & DVT & Missed diagnosis \\
2 & Kerry & 18 & PE & N/A \\
3 & Peter & 56 & DVT and PE & Family history of DVT \\
4 & Michael & 68 & PE & Family history of PE \\
5 & Sally & 48 & DVT and PE & Family history of VTE \\
6 & Rebecca & 45 & PE & Missed diagnosis \\
7 & Jenny & 63 & DVT & Speedy diagnosis \\
8 & Ralph & 63 & DVT and PE & Family history of VTE \\
9 & Eleanor & 65 & PE & Parkinson's diagnosis \\
10 & N/A & 27 & PE & ${ }^{*}$ Opted out of time two interview \\
11 & Alice & 69 & DVT and PE & Receiving chemotherapy \\
& & & & Missed diagnosis \\
12 & Joe & 68 & DVT & Missed diagnosis
\end{tabular}

*No details were forthcoming about the participant's decision to opt out of follow-up interview. DVT, venous thromboembolism; PE, pulmonary embolism; VTE, venous thromboembolism.

and experiences of diagnosis and treatment and highlighted a dual process of trauma and growth and a vicious circle of symptom-related panic. To better understand the longitudinal impact of this experience, participants were re-interviewed approximately 3 months after their first interview.

\section{METHODS}

\section{Participants}

Participants were a consecutive series of patients recruited from an out-patient haematology clinic in a UK District General Hospital. ${ }^{9}$ They were above 18 years, had experienced a first-time VTE in the 6 months prior to a first interview and consented to being re-interviewed 3 months subsequently. In total, 11 of 12 participants participated in this second interview: seven women and four men aged 18-69 (mean=53.4) years, and all white British. Participant characteristics are summarised in table 1 .

\section{Procedure}

The study was approved by an NHS Research Ethics Committee (ref: 14/WA/0019). Following written consent, participants took part in a semistructured interview, lasting between 30 and $80 \mathrm{~min}$. A semistructured interview template allowed exploration of participants' responses while ensuring that particular topics were discussed (see box 1 for interview schedule). An open and flexible interview process enabled the participants' perspectives to be accessed, allowing them to speak freely and ensuring rigour and trust worthiness of data.

\section{Patient involvement}

Patient representatives were consulted in the development of the interview topics guide and interview questions. The findings of this study will be disseminated to participants who requested this.

\section{Analysis}

Participant interviews were recorded and transcribed verbatim. Transcripts were analysed individually by RH, an experienced clinical psychologist, using inductive thematic analysis. ${ }^{10}$ This exploratory approach was best

\section{Box 1 Interview schedule}

Q1. How have things been since last time we met?Q1. How have things been since last time we met?

Q2. How would you say that the PE/DVT has affected you and your life?Q2. How would you say that the PE/DVT has affected you and your life?

Q3. Do you have to take any regular medication like an anticoagulant? Could you tell me what that's like/how you find that?Q3. Do you have to take any regular medication like an anticoagulant? Could you tell me what that's like/how you find that?

Q4. What do your family and friends think about how the PE/DVT has affected you?Q4. What do your family and friends think about how the PE/DVT has affected you?

Q5. Do you have particular worries or difficulties as a result of your PE/ DVT? These might be about physical, emotional or social?Q5. Do you have particular worries or difficulties as a result of your PE/DVT? These might be about physical, emotional or social?

Q6. Can you tell me about how you have coped since the PE/DVT?Q6. Can you tell me about how you have coped since the PE/DVT?

Q7. Finally, if you could sum up your overall experience of the DVT/PE and how it has changed or affected you and your life, what would you say?Q7. Finally, if you could sum up your overall experience of the DVT/ PE and how it has changed or affected you and your life, what would you say?

DVT, venous thromboembolism; PE, pulmonary embolism. DVT, venous thromboembolism; PE, pulmonary embolism. 
suited to elucidating the nature of a group's conceptualisation of the phenomenon under study. It has a growing body of literature examining a wide variety of health and is considered a useful method for examining different perspectives and generating novel topics insights. ${ }^{10}$ Audio recordings were listened to repeatedly to ensure accuracy and enable the identification and generation of relevant initial codes and textual units for features and patterns in the data. Extracts and phrases were used to identify potential themes, with relevant data ('quotes') gathered within identified themes. The data were systematically reviewed to ensure that a name, definition and exhaustive set of data were identified to support each category. The same unit of text could be included in more than one category. To ensure rigour and trustworthiness of recruitment, data collection and analysis, the process of saturation was adhered to. ${ }^{11}$ Themes and subthemes derived from the data and findings were checked and validated by PB; any differences were resolved through discussion.

\section{RESULTS}

Thematic analysis revealed four major themes and nine subthemes. Major themes were as follows: life changing and forever changed, the trauma of care, 'thrombo-neuroses' and through adversity comes growth.

Major theme: life changing and forever changed.

\section{A different life}

Continuing from Time One, all participants reflected on how their life had been changed by VTE and its treatment. Participants reported active attempts to integrate their experience, resulting in a new, post-VTE identity. Those taking warfarin increasingly identified as a 'warfarin patient': 'others probably don't take warfarin so they probably don't know' (Kerry), as did those around them. Their life and emerging self post-VTE were also shaped by the suddenness and life-threatening nature of the condition. It triggered reappraisal of the life lived and their future. Many reported increased spontaneity and living 'in the moment' because of the uncertainty surrounding their future health:

You really don't know what's coming next, so I think it's a good idea to do what makes you happy in that moment... It's changed me like that, so I'm more 'alright let's go!' (Kerry)

By contrast, for some, their care needs subverted the normal order of parenting, as a result of their children caring for them, or them being unable to care for their children. These experiences highlighted a loss of autonomy and purpose:

My daughter, who I frightened when I collapsed, she hovers around me... She's there every time I turn around (Eleanor)

And in the night my son would be crying and my partner...he'd be up all hours rocking him back and forth and I was just sat there watching, 'cos I couldn't do it. Physically couldn't do it. And it was really hard (Fiona)

Illness and treatment resulted in a different experience of growing up for some younger participants, differentiating them from their peer group as well as older VTE patients, leaving them isolated and highlighting the restrictions VTE and its treatment created for them:

And when it all adds up, it's like, I can't really DO anything!!...Like tattoos. I probably wouldn't ever have a tattoo but it would be nice to have the option to have it (Kerry)

\section{Changing expectations}

Participants coping responses were shaped by their changing expectations of themselves and life post-VTE. Age and life stage played an important role in this participants' adaptation. Younger participants felt it had 'brought independence a lot sooner' (Kerry, 18 years) while older participants noted an increase in dependence.

Coping resources were also influenced by their life experience and developmental stage. Younger participants reported less empathy and support from peers because they felt who did not understand VTE and its implications:

If I was older they would understand...well they might understand. But because of my age the people I socialise with don't get it, they don't get it. And they don't understand (Kerry)

By contrast, older participants acknowledged their life experiences helped them accept and integrate the experience more easily, with one commenting, "Had I been younger, it might have affected me more' (Jenny).

The anxieties relating to VTE-related symptoms reported at Time One developed, for many, into a growing awareness that symptoms and treatment may be life long. For younger patients, this realisation presented a significant physical and emotional burden and resulted in them taking increasing control over their treatment and developing a sophisticated understanding of their condition although this often went unacknowledged:

I have my INR done weekly. They [practice nurses] don't like it. But I've read evidence that weekly INR's reduce your risk by death by $46 \%$ ! They keep you in therapeutic range $90 \%$ of the time as oppose to $55 \%$ of the time when you go every 6 weeks (Fiona).

Major theme: The trauma of 'care'.

\section{Lack of care in primary care}

Consistent with the findings reported at Time One, the lack of information in relation to VTE and its treatment remained a source of frustration and resulted in several participants seeking unregulated advice on the internet. Participants reported a perceived lack of care and support in community and primary care services with negative 
interactions with primary care physicians and office staff triggering and maintaining feelings of abandonment and anxiety:

The doctors don't say to you, 'if you don't feel better come back in a couple of days'. They just give you a prescription if you've got to have one and then through the door! I've got nobody to turn to (Alice)

I think it would be better if the doctors in the surgery were more equipped to deal with the results themselves. And if they gave you the results themselves rather than the receptionist... the reception desk is right in front of where everyone is sitting! (Kerry)

Such negative experiences were most frequently reported by, but not limited to, participants who had experienced missed diagnosis and/or who were experiencing ongoing post-traumatic stress. They were anxious about symptom recurrence and believed their concerns were sometimes dismissed as inappropriate:

[I said] 'What if it's the same in 3 days? You going to refer me then?' But it was getting to the point where I was getting into a sort of argument with the doctor. And in the end I said 'don't worry, I'll ring my consultant' (Fiona).

\section{Trust: lost, broken and regained}

Participants who experienced repeated misdiagnoses reported a range of negative emotions, including blame attributed to the health professionals involved and a lack of trust in medical opinion:

It's no good going to your GP. That's one thing I'm... I'm a bit sceptical about. I think, come on! Telling me it's muscular! Twice!? (Joe)

These negative outcomes could be challenged and reversed. Rebecca, for example, described how her primary care physician made efforts to 'repair' their relationship following missed diagnosis, enabling them to rebuild trust. This contributed to a more positive experience of recovery and facilitated emotional adjustment:

[The doctor] said 'I really want to speak to you'.... And I said, 'basically I know we're all human. I said, we all make mistakes, but I'd been telling you for a very long time that I don't feel right...' And I was really nice to her cos she's a lovely, lovely woman... And I've gone back to seeing her again. And even my husband said, 'you should see someone else' and I said [to him] I'm happy with her. I said, because I know I can talk to her (Rebecca).

Major theme: Thrombo-neuroses

\section{'Post-thrombotic panic'}

Participants reported hypervigilance and panic related to fear of illness recurrence that endured over time and could be triggered by sensations associated with the experience of VTE. Some described this as living with a 'paranoia [the clots] are going to come back' (Rebecca). These anxieties formed a chronic burden, impacting on day-to-day life by contributing to episodes involving a vicious cycle of 'post-thrombotic panic' as described by one VTE patient ${ }^{8}$ (see figure 1):

Oh, if I get an ache, my one leg if I get a bit of an ache in that it's like 'oh my god, there's something happening there again' (Sally)

Anxieties were further escalated by difficulties differentiating between VTE symptoms and those of panic. Bodily sensations of breathlessness, leg pain or tightness in the chest led participants to balance the risks of attributing these sensations to panic or VTE recurrence, and whether to seek medical help:

Because it's so similar to the symptoms... as a PE that's the problem. Chest pains, can't breathe, heart racing. I don't think I will ever not be frightened of them cos no matter how much I read into them you could always have that $[\mathrm{PE}]$. There's a very small chance it could happen and you should never ignore (Kerry)

\section{The 'matrix' of anxiety and uncertainty}

Participants frequently described long-term worries and rumination focusing on the cause of their VTE and its related symptoms, including PTS:

There has to be something that's made me have this! But they can't answer that! I mean, the doctors have told me they can tell me what I haven't got, but they can't tell me what I've had (Rebecca)

Future worries related to symptom deterioration, quality of life and mortality, and reflected existential concerns about how VTE would affect participants' wider well-being as they age:

I always think about what will it be like as I get older?! As you get older you get a bit, you know, not so [healthy] as when you're younger. It does give me those thoughts then. It's like oh my god, what's my leg going to be like? And with post-thrombotic syndrome it can progress. It can get worse. It can stay the same, or it can get worse. (Fiona)

Indeed, adjusting to the permanence of post-thrombotic syndrome was a chronic source of concern for many participants. Participants were aware that VTE-related anxiety impacted on their day-to-day lives as well as their wider psychological well-being:

It's the thought that I might have to live with this. If you think you're going to get better then you just put up with it. But I suppose in the back of my mind I'm concerned that it won't improve (Eleanor)

Many found that the implications of anticoagulant treatment are restrictive and complex. The regular testing, monitoring and dosage adjustments required in warfarin treatment repeatedly triggered anxieties and 


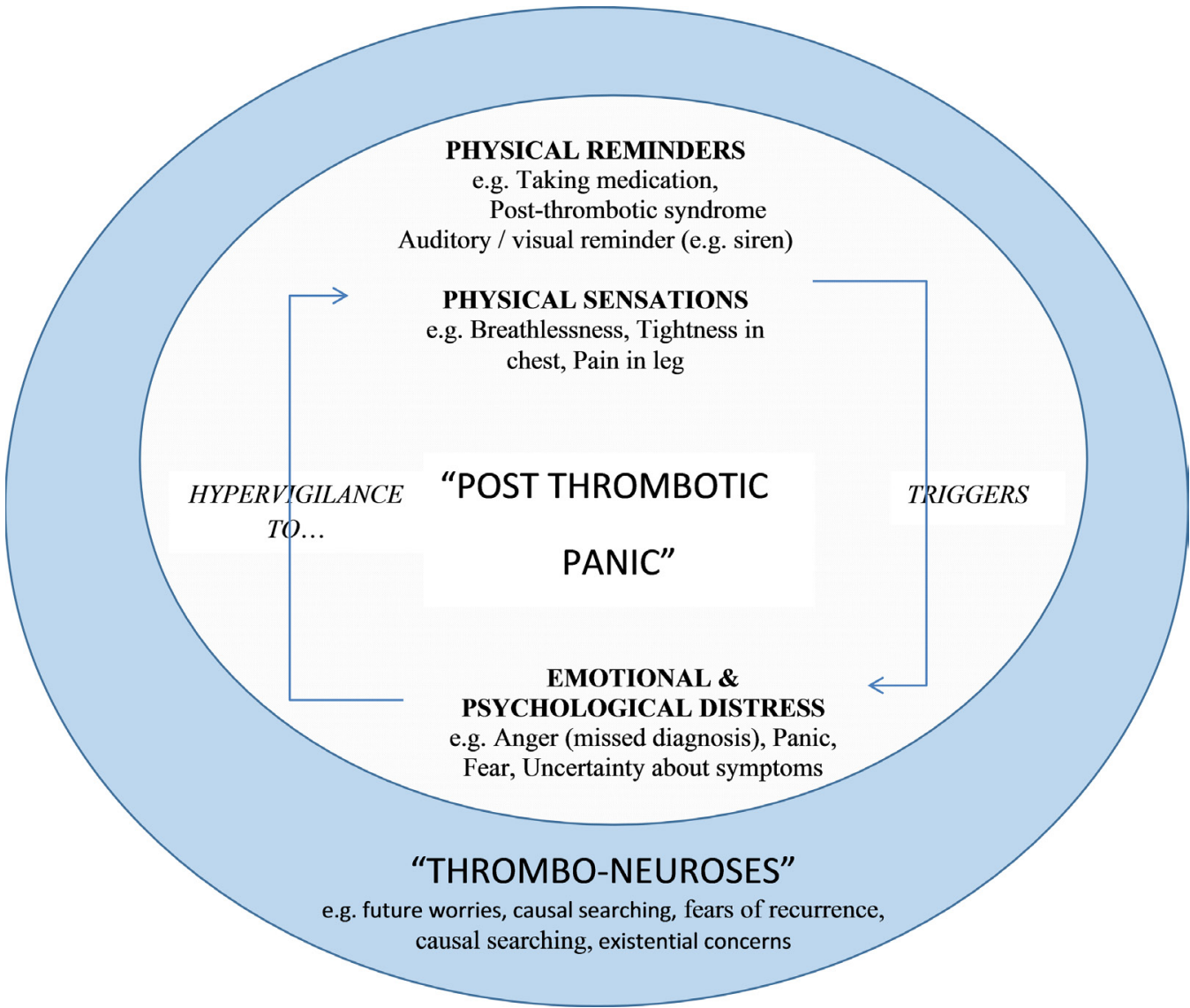

Figure 1 psychological responses to VTE including 'post-thrombotic panic' and more diffuse concerns about health and recurrence ('thrombo-neuroses'). VTE, venous thromboembolism.

memories. Some made attempts to better understand the bleeding risks and benefits of treatment, which led to further anxieties:

It's not just black and white. It is kind of...that's one thing, but there's about twelve branches off it that could happen. And then that's another thing that has... It's a bit like the Matrix, you take one pill or the other pill and the whichever one you take... [shrugs] (Kerry)

\section{A private struggle with emotion}

Most participants reported being reluctant to share their worries with health professionals, unwilling to appear ungrateful to the healthcare system. They were also concerned about burdening family and friends, felt unable to fully articulate their concerns or believed they would not be fully understood:

I'm not saying he's said he [husband] doesn't want to hear it but I wouldn't expect him to sit through. He couldn't sit there and listen to me talking about it (Sally)

Participants commented that "nobody talked about the psychological side of things' (Eleanor) and so the struggle with emotions remained hidden, exacerbating anxieties and preventing access to appropriate support. These private worries were separate from the more acute cycle of hypervigilance and panic and were chronic, repetitive, and involved a dual awareness and avoidance of worries:

There's probably an underlying worry about will it happen again, and all that nonsense. But I try to put it right at the back of my mind (Michael)

I don't think a great deal about it. It will always be in the back of your head (Ralph)

By contrast, most participants felt that being able to discuss their fears and engage with fellow patients could have been of benefit. The likely extent of these various emotion difficulties may be determined from, as yet, unpublished data from the larger longitudinal study from which participants were recruited, in which $36 \%$ reported high levels of post-trauma symptomology (score of 24 or more on the Impact of Events scale-revised), ${ }^{8}$ $22 \%$ met the criteria for 'probable diagnosis of post-traumatic stress disorder' and 39\% had 'severe' psychological morbidity as assessed by the Hospital Anxiety and Depression Scale12. ${ }^{9}$

Major theme: Through adversity comes growth

\section{Cumulative adversity: the hero's journey}

By the present interviews, the realisation that VTE-related physical difficulties may endure or worsen over 
time had become more evident to participants over time. Recovery presented enduring physical and psychological challenges, and coping with or overcoming them enabled participants to grow in confidence, and contributed to a search for meaning:

So that's been the benefits of having a pulmonary embolism, so to speak [laughs]. It's focussed my mind to lose weight and just think about healthy lifestyles. In a way I probably wasn't beforehand. Wake-up call that's what you call it (Peter)

Their experiences focused participants on their strengths, which began to define their emerging post-VTE identity and enabled a view of their illness experience through the lens of a survivor who had overcome adversity:

I think I could deal with anything now really! throw the book at me! [laughs] (Rebecca)

I think I'll do things more. I know that sounds silly. Because it's stopped me from doing a lot of things (Kerry)

The parental role, in particular, highlighted a duality of negative and positive impacts of the VTE experience. Parenting provided a reason greater than oneself for which to overcome the challenges faced post-VTE:

Like my partner said, 'there's no chance of the boys [sons] ever having anything wrong with them because you'll make sure they won't!' [laughs]. And yeah, that's good. And I help everybody who's in my life (Fiona)

The duality was also reflected in descriptions of benefits and growth as being inter-related. The impact of living with VTE-related uncertainty led participants to search for meaning in their experience and this, in turn, enabled them to find positives:

You know, in some ways it has been difficult. It's a whole 'pull you up' incredibly sharp and think about your life. But emerging out of that there's been the value of taking health more seriously (Peter)

You'd almost say it's done me good (Michael)

\section{'Enlightenment'}

Even more evident than in Time One interviews, many participants described aspects of the VTE and their response as an existential experience that 'really opened my eyes' (Michael). Participants likened this to 'enlightenment' and described how things 'just sort of clicked' (Kerry) and the 'mist clears' (Rebecca) in a way that facilitated adjustment:

Well things just seem to fall into place. The slightest thing. I can't really explain it. It's like an enlightenment. It's like, ping! Something goes off in your brain and you think, 'oh god it's really not as bad as that!' (Sally)
Participants reflected on their life in general and engaged in reprioritisation leading to active changes in their life, such as weight loss, spending savings and changing personal relationships. Central to that was the life-threatening nature of VTE which served as both catalyst and maintaining factor for these post-VTE alterations:

Oh yeah. You're out to enjoy your life now and get what you can out of it. It's something you don't really think about - death (Ralph)

Life's short, live it to the full as much as you can afford to do so. And have time to do so. That's been drummed into me for god knows how long but I'm only now beginning to realise (Michael)

\section{DISCUSSION}

This study followed up 11 participants, previously interviewed within the first 6 months of a first-time VTE, about its psychosocial impact ${ }^{9}$ over the subsequent 3 months. This time frame was considered significant as participants had now moved beyond initial illness and diagnosis and many decisions had been made about their longer-term management of care, in particular whether they would remain on anticoagulation treatment. Some issues remained constant. As at Time One, participants reported significant levels of anxiety with many experiencing ongoing symptoms of trauma including intrusive thoughts, flashbacks and hypervigilance. The previously reported cycle of 'post-thrombotic panic' remained, and participants continued to experience a vicious circle of panic and hypervigilance maintained by VTE-related symptoms (see figure 1). The hypervigilance to bodily symptoms, common following illness, ${ }^{12}$ reflected the difficulties participants faced in avoiding internal sensations and reminders of the VTE and differentiating between symptoms of anxiety and VTE (eg, shortness of breath, leg pain, tightness in chest), with implications for overuse of healthcare resources as well as individual distress.

Negative emotions, such as anger and sadness, experienced because of missed diagnosis and chronic VTE-related symptoms (including post-thrombotic syndrome, pain, etc), notably remained a concern for participants. They precipitated and perpetuated intrusive memories and anxieties. Furthermore, these emotions negatively impacted on relationships with professionals and influenced the use of healthcare resources. Anticoagulant treatment with warfarin necessitates regular monitoring to manage the risk of haemorrhage and this provided frequent reminders of the VTE, treatment restrictions and future health risk; all of which contributed to low mood and anxiety. Participants also continued to experience wider, more diffuse fears of VTE recurrence, causal searching and concerns about future health alongside worries and restrictions about anticoagulation treatment. These anxieties may be understood as wider 'thrombo-neuroses' and weighed heavily on participants, 
described metaphorically as 'the sword of Damocles' or 'a dark cloud' with which they must learn to live.

This study also highlights other potential subgroups of VTE patients that may require additional support. Younger VTE patients may be most at risk of illness -related isolation and health-related anxiety due to greater levels of uncertainty when contemplating their future, and they may be further disadvantaged by a lack of the life experience and resources on which older people can draw. ${ }^{13}$ As such, it is important to consider that VTE may impact a young person's development, with consequences for their emerging sense of identity. Finally, the findings highlight the continuation of a dual process of trauma and growth in the aftermath of VTE, which may be best understood as post-trauma growth and benefit finding, ${ }^{14}$ both of which have been reported following a range of physical health disorders. ${ }^{15}$ More definitive evidence of these processes in VTE could be usefully determined by larger, quantitative, studies.

In considering the limitations of this research, it is important to acknowledge the relatively small number of participants in the study, and the potential bias as a consequence of self-selection which limits interpretation and generalisability of the findings. More studies with larger populations are clearly required. Nevertheless, the study provides the first longitudinal in-depth study of the experience of this group of patients. The findings support those reported in unrepresentative samples which suggest that peri-traumatic factors seem to play a key role in the development and maintenance of post-traumatic responses. ${ }^{5}$ Furthermore, it seems that through coping with such adversities, individuals are able to experience growth, lending weight to the suggestion that the consequence of a traumatic illness onset is multidimensional, encompassing both negative and positive aspects. ${ }^{14} 15$ In particular, it supports the notion that self-perceived post-traumatic growth is both a coping effort and a consequence of coping. ${ }^{16}$

Future research may usefully compare the relationship between anxiety, health concerns, and warfarin treatment and its frequent engagement with health professionals, with the newer direct acting oral anticoagulant (DOAC) treatments which require lower levels of monitoring. The dynamics between the reassurance of drug levels being closely monitored at the cost of frequent reminders of patients' condition and lower levels of monitoring, with less potentially reassuring data are important from a clinical perspective. As a cautionary note, since completing the study we have been made aware of 'warfarin envy', a state in which individuals taking DOACs prefer the constant monitoring of warfarin levels to their absence. Given these psychological responses to differing treatment approaches, it would be of value to determine what type of medication regimen suits which individuals. Relatedly, it would be beneficial to understand how younger VTE patients and those affected by missed diagnosis are disadvantaged compared with older VTE patients and those with a clearer diagnostic pathway.
Finally, it is important to consider how targeted support can help patients to manage their recovery ${ }^{17}$ in such a way as to facilitate growth. ${ }^{9}$ Cognitive behavioural therapy protocols for anxiety disorders have traditionally aimed to modify faulty threat appraisals and their value can be questioned in a population for whom health risks are a real threat. However, cognitive acceptance and mindfulness-based interventions may clearly be of benefit. ${ }^{18} 19$

Contributors SN, PB, SL, RH conceived the study. RH undertook the interviews. RH and PB undertook the analysis of data. All authors contributed to the final version of the manuscript.

Funding The research was completed as part of a PhD funded by Thrombosis Research in Advanced Disease.

Competing interests None declared.

Patient consent for publication Not required.

Ethics approval Ethical approval was obtained through the National Health Service (NHS) South Wales Ethics Committee.

Provenance and peer review Not commissioned; externally peer reviewed.

Data sharing statement Additional data are available upon reasonable request from the corresponding author.

Open access This is an open access article distributed in accordance with the Creative Commons Attribution Non Commercial (CC BY-NC 4.0) license, which permits others to distribute, remix, adapt, build upon this work non-commercially, and license their derivative works on different terms, provided the original work is properly cited, appropriate credit is given, any changes made indicated, and the use is non-commercial. See: http://creativecommons.org/licenses/by-nc/4.0/.

\section{REFERENCES}

1. Beckman MG, Hooper WC, Critchley SE, et al. Venous thromboembolism: a public health concern. Am J Prev Med 2010;38:S495-S501.

2. White RH. The epidemiology of venous thromboembolism. Circulation 2003;107:41-8.

3. Heit JA, Silverstein MD, Silverstein MD, et al. Predictors of recurrence after deep vein thrombosis and pulmonary embolism: a population-based cohort study. Arch Intern Med 2000;160:761-8.

4. Yamaki T, Hamahata A, Soejima K, et al. Factors predicting development of post-thrombotic syndrome in patients with a first episode of deep vein thrombosis: preliminary report. Eur J Vasc Endovasc Surg 2011;41:126-33.

5. Bennett P, Patterson K, Noble S. Predicting post-traumatic stress and health anxiety following a venous thrombotic embolism. J Health Psychol 2016;21:863-71.

6. Kahn SR, Ducruet T, Lamping DL, et al. Prospective evaluation of health-related quality of life in patients with deep venous thrombosis. Arch Intern Med 2005;165:1173-8.

7. van Es J, den Exter PL, Kaptein AA, et al. Quality of life after pulmonary embolism as assessed with SF-36 and PEmb-QoL. Thromb Res 2013;132:500-5.

8. Katon W, Lin EH, Kroenke K. The association of depression and anxiety with medical symptom burden in patients with chronic medical illness. Gen Hosp Psychiatry 2007;29:147-55.

9. Hunter R, Lewis S, Noble S, et al. "Post-thrombotic panic syndrome": A thematic analysis of the experience of venous thromboembolism. Br J Health Psychol 2017;22:8-25.

10. Braun V, Clarke V. Using thematic analysis in psychology. Qual Res Psychol 2006;3:77-101.

11. O’Reilly M, Parker N. "Unsatisfactory Saturation": a critical exploration of the notion of saturated sample sizes in qualitative research. Qualitative Research 2013;13:190-7.

12. Weiss DS. The impact of event scale: revised. Cross-cultural assessment of psychological trauma and PTSD. Boston, MA: Springer, 2007:219-38.

13. Corbeil A, Laizner AM, Hunter P, et al. The experience of uncertainty in young adults with cancer. Cancer Nurs 2009;32:E17-E27.

14. Joseph S. What doesn't kill us: The new psychology of posttraumatic growth. New York: Basic Books, 2011.

15. Hefferon K, Grealy M, Mutrie N. Post-traumatic growth and life threatening physical illness: a systematic review of the qualitative literature. Br J Health Psychol 2009;14:343-78. 
16. Maercker A, Zoellner T. The Janus face of self-perceived growth: Toward a two-component model of posttraumatic growth. Psychological Inquiry 2004;15:41-8.

17. Noble S, Lewis R, Whithers $\mathrm{J}$, et al. Long-term psychological consequences of symptomatic pulmonary embolism: a qualitative study. BMJ Open 2014;4:e004561.
18. Lundgren T, Dahl J, Hayes SC. Evaluation of mediators of change in the treatment of epilepsy with acceptance and commitment therapy. $J$ Behav Med 2008;31:225-35.

19. Montgomery KL, Kim JS, Franklin C. Acceptance and commitment therapy for psychological and physiological illnesses: a systematic review for social workers. Health Soc Work 2011;36:169-81. 